\title{
Active Rebleeding of a Ruptured Middle Cerebral Artery Aneurysm During Diagnostic Catheter Angiography
}

\author{
Pinar CELTIKCl ${ }^{1}$, Onder ERASLAN ${ }^{1}$, Onur ERGUN¹, Esra SOYER GULDOGAN ${ }^{1}$, Mehmet Erhan TURKOGLU² \\ 'Diskapi Yildirim Beyazit Training and Research Hospital, Radiology Clinic, Ankara, Turkey \\ 2Diskapi Yildirim Beyazit Training and Research Hospital, Neurosurgery Clinic, Ankara, Turkey \\ This study has been presented at the $37^{\text {th }}$ National Radiology Congress between 01 and 05 November 2016 at Antalya, Turkey.
}

To watch the surgical videoclip, please visit http://turkishneurosurgery.org.tr/images/19629_video.mp4

\section{ABSTRACT}

Rebleeding of cerebral aneurysms has been previously reported during diagnostic angiography. However, capturing the exact moment of active rebleeding of a cerebral aneurysm during angiography is extremely rare. Here, a case of a rebleeding middle cerebral artery aneurysm during diagnostic digital subtraction angiography (DSA) was illustrated, accompanied with a video demonstration of the incident which is the only one in the literature. During the acquisition of lateral projection DSA images, active extravasation of the contrast medium was witnessed, indicating rebleeding. Simultaneously, there was a sudden rise in arterial blood pressure and an episode of bradycardia. The procedure was terminated immediately and the patient was transferred to the intensive care unit for extraventricular drainage and stabilization of vital signs. Unfortunately, the patient was lost.

KEYWORDS: Aneurysm, Cerebral angiography, Complication, Rebleeding, Subarachnoid hemorrhage

ABBREVIATIONS: ABP: Arterial blood pressure, CT: Computed tomography, DSA: Digital subtraction angiography, GCS: Glasgow Coma Scale, ICA: Internal carotid artery, MCA: Middle cerebral artery, SAH: Subarachnoid hemorrhage.

\section{INTRODUCTION}

$\mathrm{T}$ The most common cause of subarachnoid hemorrhage $(\mathrm{SAH})$ is a ruptured cerebral aneurysm, approximately in $85 \%$ of the cases (18). Cerebral catheter angiography is a commonly utilized imaging technique, in order to detect and define cerebral aneurysms in terms of size, shape and location preoperatively.

Rebleeding of aneurysms during diagnostic angiography was previously reported. However, capturing the exact moment of active rebleeding of a cerebral aneurysm during angiography is extremely rare $(6,12)$. Here, we illustrate a case of active rebleeding from a left middle cerebral artery (MCA) aneurysm that was captured during diagnostic digital subtraction angiography (DSA).

\section{CASE REPORT}

An 85-year-old female patient was admitted to emergency unit with loss of consciousness. Patient history was significant for hypertension. Glasgow Coma Scale (GCS) score at the initial examination was 5 (no eye opening, no verbal response, abnormal flexion). Computed tomography (CT) revealed extensive SAH predominantly in the left Sylvian fissure and sulci of frontal, temporal and parietal lobes of the left hemisphere accompanied by a hematoma $7 \times 6 \mathrm{~cm}$ in size and 
midline shift (Video 1). A nodular density of $10 \mathrm{~mm}$ in diameter was noted at the level of left MCA, suggestive of an aneurysm. Following neurosurgical evaluation, preoperative diagnostic cerebral DSA was ordered. Arterial blood pressure (ABP) of the patient was $160 / 100 \mathrm{~mm} \mathrm{Hg}$ when the procedure was initiated. As the SAH was more extensive on the left side, left internal carotid artery (ICA) was catheterized initially. Towne projection (anteroposterior projection with a $30^{\circ}$ craniocaudal angle) revealed a saccular aneurysm, $13 \times 10 \mathrm{~mm}$ in size, at the MCA bifurcation. During the acquisition of lateral projection DSA images, active extravasation of the contrast medium was witnessed indicating rebleeding (Figure 1A-D and Video 1). Simultaneously, there was a sudden rise of ABP to $220 / 120 \mathrm{~mm} \mathrm{Hg}$ and an episode of bradycardia occurred. Procedure was terminated immediately and the patient was transferred to intensive care unit for extraventricular drainage and stabilization of vital signs. GCS score was 3 at the time with bilateral fixed and dilated pupils. During extraventricular drainage, there was a sudden drop of ABP, followed by a cardiac arrest, and the patient unfortunately died.

\section{DISCUSSION}

Recurrent bleeding is the most feared complication of a cerebral aneurysm with mortality rates of $50-80 \%$ (6). This complication occurs more frequently in the first 24 hours of the aneurysmal rupture (9). Rebleeding of a cerebral aneurysm during diagnostic catheter angiography was described first by Abbott et al. in 1952 (1). In the literature, the incidence was reported between $0.01-4 \%(9,11,15)$. This entity is more common among female patients which could be secondary to female predisposition for SAH $(2,5,11,19)$. More recent publications reported rebleeding during head CT angiography as well $(3,13)$.

Poor neurological condition at admission, aneurysm site and angiography within the first 6 hours of SAH were proposed as risk factors for rebleeding. Early angiography appears to be a prominent risk factor according to previous publications $(2,10,19)$. Komiyama et al. reviewed 202 cases from the literature including 14 cases of their own. Their results demonstrated that the majority of rebleeding incidents during angiography had occurred in the first day of SAH and $89 \%$ of them bled when the angiography was performed in the first 6 hours (9). Saitoh et al. found that angiography within the 6 hours of initial SAH was associated with a significantly higher incidence of rebleeding (15). Kusumi et al. reported that re-rupture during angiography was more common when angiography was obtained in the first 3 hours (11). Increased risk of rebleeding during angiography in this early period could

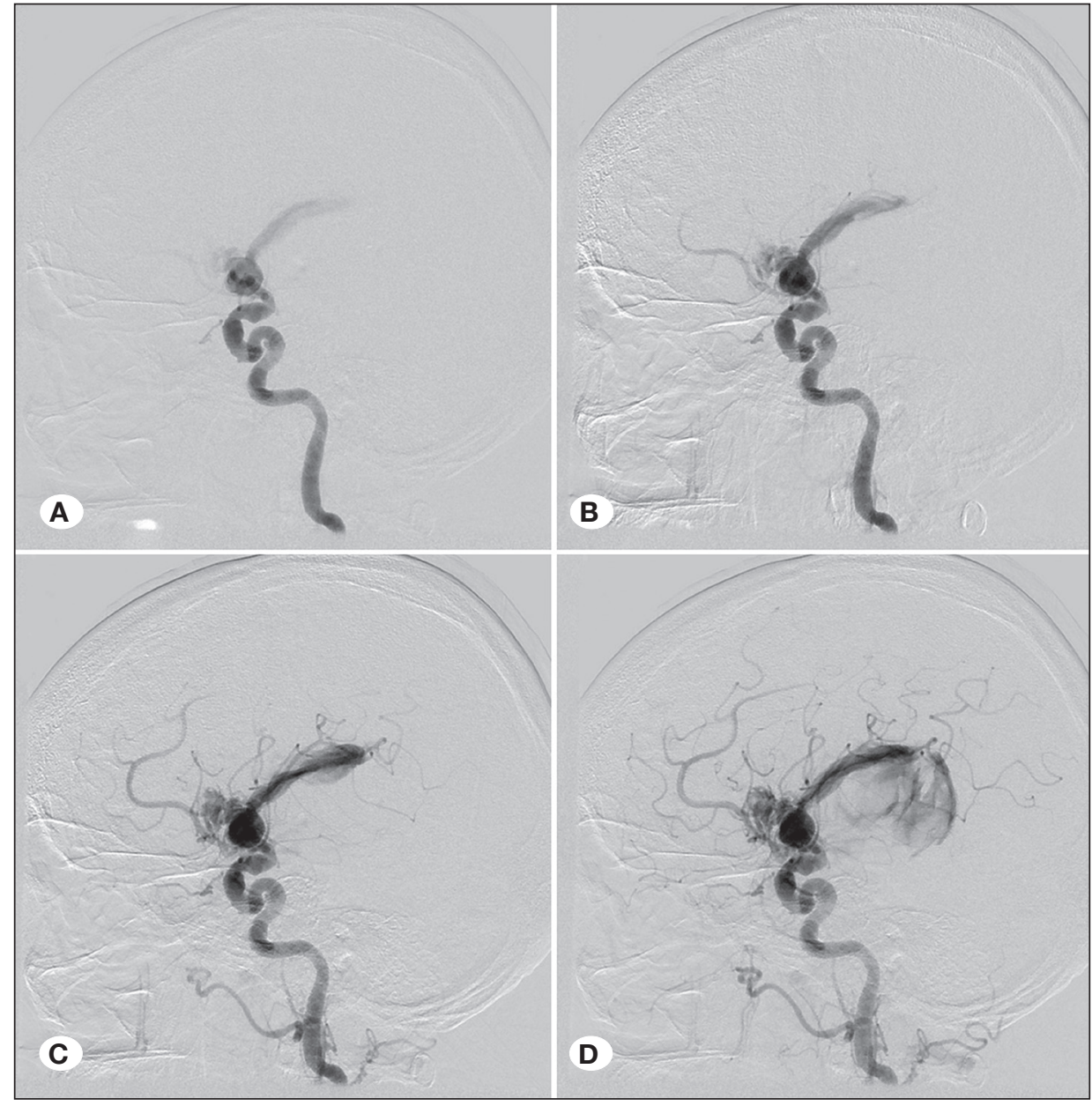

Figure 1: Subsequent cerebral DSA images in lateral projection demonstrating active extravasation from the rebleeding left MCA aneurysm (A, B, C, D). 
be secondary to relatively fragile hemostatic plug at the rupture site. Therefore, some authors advocate avoiding angiography studies in the first 6 hours of SAH whenever possible $(2,9,11)$. In literature, the majority of the rebleeding aneurysms during angiography were located at the ICA or MCA $(2,6,9,11,19)$. The reason behind this tendency could be the higher incidence of aneurysms located at the anterior circulation. In addition, some authors suggested that the abrupt intraluminal pressure changes in this two arteries due to their proximity to the contrast injection site combined with the lack of support from the surrounding brain parenchyma due to cisternal location might play a role $(2,9)$. Poor neurological condition on arrival was also proposed as a risk factor (3).

The mechanism of this complication whether incidentally or secondary to the angiography procedure remains unsolved. There is some evidence suggesting the injection of contrast medium causes an increase in the intracarotid pressure and subsequently intraaneurysmal pressure $(4,14,16,17)$. An intracranial Doppler ultrasonography study suggested that cerebral angiography may affect the tonus of the cerebral vessels in a minority of patients, mostly in the form of vasodilation, which might be a factor for rebleeding as well (7). Another study suggested to reduce the injection rate of the contrast material during imaging in order to prevent the sudden increase of the intraluminal pressure $(2,8,14)$. Abrupt rise of intraluminal pressure was significantly more prominent with conventional angiography compared to DSA (2). Advances in the angiography technique (conventional cervical puncture to transfemoral DSA and automatic injectors) and introduction of less irritant contrast media might have provided fewer occurrence of this complication $(8,9)$. Although, rebleeding of a cerebral aneurysm has been linked to elevated systolic blood pressure, no direct relation to rebleeding during angiography was reported (11). Still, tight ABP control prior to the angiography procedure could prevent the effect of contrast material injection on the vulnerable rupture site.

The management of a rebleeding aneurysm consists of blood pressure control accompanied with extraventricular drainage. Operation could be considered following rebleeding provided that the patient condition is stable enough.

To the best of our knowledge, our case illustration is the only one in the literature that includes a video demonstration of the rebleeding moment. Our case illustrates the main risk factors for this complication. Patient was an elderly female, with a saccular aneurysm located at MCA and poor neurological condition at arrival. As the patient was found unconscious, the exact time interval between the insult and DSA was unclear. However, the interval between hospital admittance and DSA was shorter than 3 hours. The borderline high ABP at the beginning of the procedure $(160 / 100 \mathrm{~mm} \mathrm{Hg})$ might have been a precipitating factor as well. However, the presence of a large hematoma secondary to first rupture and the midline shift required an urgent DSA prior to the emergency surgery.

\section{CONCLUSION}

With the guidance of this case illustration and as advised in the literature, we suggest that, diagnostic angiography might be avoided in the first 6 hours of SAH and ABP control should be achieved during this period, in order to prevent rebleeding during angiography. However, as it was the case in our patient, angiography could not be delayed safely when urgent surgical intervention is warranted. Both the interventional radiologist and the neurosurgeon should be aware of the risk factors related to this complication and avoid them whenever possible.

\section{- REFERENCES}

1. Abbott KH, Gay JR, Goodall RJ: Clinical complications of cerebral angiography. J Neurosurg 9: 258-274, 1952

2. Aoyagi N, Hayakawa I: Rerupture of intracranial aneurysms during angiography. Acta Neurochir (Wien) 98: 141-147, 1989

3. Hashiguchi A, Mimata $\mathrm{C}$, Ichimura $\mathrm{H}$, Morioka $\mathrm{M}$, Kuratsu $\mathrm{J}$ : Rebleeding of ruptured cerebral aneurysms during threedimensional computed tomographic angiography: Report of two cases and literature review. Neurosurg Rev 30: 151-154, 2007

4. Hinshaw DB, Simmons CR, Leech W, Minckler J, Austin G: Loculated intracranial aneurysms: Angiography and possible etiology. Radiology 113: 101-106, 1974

5. Inagawa T, Kamiya K, Ogasawara H, Yano T: Rebleeding of ruptured intracranial aneurysms in the acute stage. Surg Neurol 28: 93-99, 1987

6. Klisch J, Weyerbrock A, Spetzger U, Schumacher M: Active bleeding from ruptured cerebral aneurysms during diagnostic angiography: Emergency treatment. AJNR Am J Neuroradiol 24: 2062-2065, 2003

7. Kochanowicz J, Lewszuk A, Kordecki K, Swiercz M, Mariak $Z$ : Diagnostic cerebral angiography affects the tonus of the major cerebral arteries. Med Sci Monit 13 Suppl 1: 55-58, 2007

8. Koenig GH, Marshall WH, Poole GJ, Kramer RA: Rupture of intracranial aneurysms during cerebral angiography: Report of ten cases and review of the literature. Neurosurgery 5: 314324, 1979

9. Komiyama M, Tamura K, Nagata $\mathrm{Y}, \mathrm{Fu} \mathrm{Y}$, Yagura H, Yasui T: Aneurysmal rupture during angiography. Neurosurgery 33 : 798-803, 1993

10. Kuhn J, Vehlen C, Mennel HD, Mahkorn D, Bewermeyer $\mathrm{H}$ : Rupture of an internal carotid artery aneurysm during angiography with leakage of contrast medium via an external ventricular drain. Neuroradiology 45: 905-907, 2003

11. Kusumi $M$, Yamada $M$, Kitahara $T$, Endo $M$, Kan $S$, lida $H$, Sagiuchi T, Fujii K: Rerupture of cerebral aneurysms during angiography-a retrospective study of 13 patients with subarachnoid hemorrhage. Acta Neurochir (Wien) 147: 831837, 2005

12. Liliequist $B$, Lindqvist $M$, Probst F: Rupture of intracranial aneurysm during carotid angiography. Neuroradiology 11: 185-190, 1976 
13. Nakatsuka M, Mizuno S, Uchida A: Extravasation on three-dimensional CT angiography in patients with acute subarachnoid hemorrhage and ruptured aneurysm. Neuroradiology 44: 25-30, 2002

14. Saitoh H, Hayakawa K, Nishimura K, Okuno Y, Murayama C, Miyazawa T, Zieroth BF, Shimizu Y: Intracarotid blood pressure changes during contrast medium injection. AJNR Am J Neuroradiol 17: 51-54, 1996

15. Saitoh H, Hayakawa K, Nishimura K, Okuno Y, Teraura T, Yumitori K, Okumura A: Rerupture of cerebral aneurysms during angiography. AJNR Am J Neuroradiol 16: 539-542, 1995
16. Sorimachi T, Takeuchi S, Koike T, Minakawa T, Tanaka R: Intra-aneurysmal pressure changes during angiography in coil embolization. Surg Neurol 48: 451-457, 1997

17. Stoeter P, Prey N, Hoffmann C, Büdingen HJ, Bergleiter R: Doppler-sonographic examination of the arterial flow in the carotid and supratrochlear arteries during carotid angiography. Neuroradiology 26: 199-207, 1984

18. Van Gijn J, Rinkel GJ: Subarachnoid haemorrhage: Diagnosis, causes and management. Brain J Neurol 124: 249-278, 2001

19. Zaehringer M, Wedekind C, Gossmann A, Krueger K, Trenschel G, Landwehr P: Aneurysmal re-rupture during selective cerebral angiography. Eur Radiol 12 Suppl 3: S18-24, 2002 